\title{
A Novel Approach for Optimized Test Case Generation using Activity and Collaboration Diagram
}

\author{
Baikuntha Narayan Biswal \\ National Institute of Technology \\ Rourkela, India
}

\author{
Soubhagya Sankar Barpanda \\ National Institute of Technology \\ Rourkela, India
}

\author{
Durga Prasad Mohapatra \\ National Institute of Technology \\ Rourkela, India
}

\begin{abstract}
Testing is the process of building confidence of the programmer that shows, the software does what it is intended to do, which in turn improves the reliability of the software. And automation of software testing process helps in achieving it with reduced cost and time. Test case generation is one part of the testing process with description of a test and independent of designed system, intended to find errors. The advantage of generation of test cases from specifications and design is that they can be available during early phase of the software development life cycle and there is no need to wait for development of codes to test the software. Additionally early test case generation reduces errors, inconsistencies and ambiguities, during the life cycle, because developers can use test cases to control their program to conform to the software specification. In this paper we have applied Constraint-based Genetic Algorithm technique to generate optimized test cases from UML Activity diagram and Collaboration diagram. Our proposed approach is more effective, which uncovers more number of errors, by using combinatorial optimization technique such as genetic algorithm with transition coverage, a test adequacy criterion as a constraint. We have defined an error minimization technique in our approach, which works as a basic principle for optimized test case generation. That means the generated test case have lower chance of presence of errors, by discarding the rest. The proposed approach is discussed by considering ATM cash withdrawal as a case study.
\end{abstract}

The full text of the article is not available in the cache. Kindly refer the IJCA digital library at www.ijcaonline.org for the complete article. In case, you face problems while downloading the full-text, please send a mail to editor at editor@ijcaonline.org 